Nervenarzt 2016 · [Suppl 1]: 87:S1 DOI 10.1007/s00115-016-0139-4

Online publiziert: 20. Juni 2016

๑) Springer-Verlag Berlin Heidelberg 2016

CrossMark

Die Deutsche Gesellschaft für Neurologie (DGN) ist mit der bisherigen Aufarbeitung der für sie relevanten Geschehnisse während der Zeit des Nationalsozialismus (NS) nie vollkommen zufrieden gewesen. Über 70 Jahre nach dem Ende des NS-Regimes sind viele Fragen zur Rolle der Neurologie und deren Protagonisten in dieser Zeit nicht ausreichend beantwortet.

Daher hat die DGN Ende 2014 einen Forschungsauftrag mit dem Ziel einer wissenschaftshistorischen Analyse zu eben diesem Thema vergeben. Bei Sichtung der in der vorliegenden Publikation dargestellten Resultate kommen - trotz aller Genugtuung über die erzielten Fortschritte - naturgemäß weitere Anliegen oder auch Wünsche auf, welche durchaus in einen langjährigen und lebhaften Kontakt mit den Medizinhistorikern einmünden könnten.

Wir sind es den Opfern der NS-Zeit schuldig, etwa die Lebenswege der vertriebenen Wissenschaftler und auch der niedergelassenen Kolleginnen und Kollegen nachzuzeichnen und ans Licht $\mathrm{zu}$ bringen, um uns einem kompletten Vergessen entgegenzustellen - eine sehr aufwendige und lange andauernde Arbeit.

Auch die Biografien derjenigen Neurologen möchten wir wissenschaftlich erschließen, deren Rolle und Einfluss in der Vergangenheit nicht eindeutig bewertet werden konnten, sei es weil Archivmaterial nicht bekannt oder zugänglich war, sei es weil der geringere zeitliche Abstand zu den Geschehnissen eine distanziertere Sichtweise erschwerte. Solche Nachforschungen sind jedoch unbedingt erforderlich, bevor man sich zum Beispiel mit nach bekannten Persönlichkeiten der Neurologie benannten Preisen oder In-

\author{
M. Grond ${ }^{1} \cdot$ T. Thiekötter ${ }^{2}$ \\ ${ }^{1}$ Klinik für Neurologie, Kreisklinikum Siegen $\mathrm{GmbH}$, Siegen, Deutschland \\ ${ }^{2}$ Deutsche Gesellschaft für Neurologie, Berlin, Deutschland
}

\title{
Dem Vergessen entgegenstellen
}

stitutionen, aber auch mit dem Gebrauch belasteter Eponyme ernsthaft auseinandersetzen kann.

\section{) Wichtige Erkenntnisse wurden erst durch eine zeitliche Distanz möglich}

Diese Wünsche werden nicht zuletzt verstärkt durch die in diesem Heft klar herausgearbeitete Erkenntnis, dass oft Wissenschaftler sowie Ärztinnen und Ärzte aller Couleur nicht wehrlose Opfer des NS-Regimes waren, sondern dass sich Regime und Mediziner durchaus gegenseitig (in einer klassischen Win-win-Situation) von Nutzen waren: hier aktive Unterstützung und wissenschaftlich ,untermauerte" Rechtfertigung einer Ideologie (mit den bekannten Folgen), dort im Gegenzug Bereitstellungvon Ressourcen, Macht, Ansehen, Bedeutung für den Einzelnen wie auch seine jeweilige Institution. Dies bedeutet implizit, dass die Akteure wissen mussten, in welchem Kontext sie sich beruflich bewegten, woraus wiederum folgt, dass sie eine Wahl hatten, Dinge zu tun oder - unter Inkaufnahme gewichtiger Nachteile und sogar Gefahren - zu lassen. Auch diese wichtige Erkenntnis wurde erst durch eine zunehmende zeitliche Distanz zum damaligen Geschehen sowie dessen aktiver Aufarbeitung auf verschiedenen Ebenen möglich und schlägt sich in den Folgegenerationen in einer grundsätzlich veränderten Einstellung zum Geschehen nieder.

Diesen Impuls möchten wir nutzen, um in unserem Bereich tiefere Kenntnisse zu gewinnen, „Aufarbeitungslücken“ zu schließen, Verdrängungsprozesse auf- zulösen und damit ein wenig dazu beizutragen, dass uns und den folgenden Generationen „Solches“ erspart bleibe.

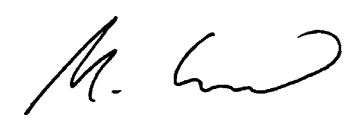

Prof. Dr. Martin Grond
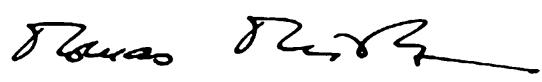

Dr. Thomas Thiekötter

\section{Korrespondenzadresse}

\section{Dr. T. Thiekötter}

Deutsche Gesellschaft für Neurologie Reinhardtstr. 27 C, 10117 Berlin, Deutschland

thiekoetter@dgn.org

Danksagung. Unser Dank gilt den Autoren dieses Heftes für deren anspruchsvolle, uns sehr wertvolle Arbeit, dem Präsidium der DGN für rückhaltlose und engagierte Unterstützung dieses Projekts, Prof. Otto Busse für seine kundige und engagierte Beratung sowie Prof. Chr. Gerloff und Prof. T. Back für ihre hilfreichen und weiterweisenden Kommentare; dem Springer Verlag Dank für die vorbildliche Umsetzung des Werks.

Interessenkonflikt. M.Grond und T. Thiekötter geben an, dass kein Interessenkonflikt besteht. 